\title{
On tensor products of irreducible integrable representations
}

\author{
Shifra Reif ${ }^{a, *, 1}$, R. Venkatesh ${ }^{\mathrm{b}, 2}$ \\ a Bar-Ilan University, Ramat Gan, Israel \\ b Indian Institute of Science, Bangalore, India
}

\section{A R T I C L E I N F O}

Article history:

Received 21 December 2020

Available online 15 November 2021

Communicated by Volodymyr

Mazorchuk

\section{Keywords:}

Borcherds-Kac-Moody algebras

Integrable representations

Unique factorization

Kac-Weyl character formula

\section{A B S T R A C T}

We consider integrable category $\mathcal{O}$ representations of Borcherds-Kac-Moody algebras whose Cartan matrix is finite dimensional, and determine the necessary and sufficient conditions for which the tensor product of irreducible representations from this category is isomorphic to another. This result generalizes a fundamental result of C. S. Rajan on unique factorization of tensor products of finite dimensional irreducible representations of finite dimensional simple Lie algebras over complex numbers.

() 2021 Published by Elsevier Inc.

\footnotetext{
* Corresponding author.

E-mail addresses: shifra.reif@biu.ac.il (S. Reif), rvenkat@iisc.ac.in (R. Venkatesh).

1 SR was partly funded by ISF Grant No. 1221/17 and ISF Grant No. 1957/21.

2 RV was partially funded by the grants DST/INSPIRE/04/2016/000848, MTR/2017/000347 from DST (Govt. of India), F.510/25/CASII/2018(SAP-I) from UGC (Govt. of India) and an Infosys Young Investigator Award.
} 


\section{Introduction}

A theorem of Rajan [11] asserts the following fundamental property of tensor products of irreducible representations of a finite dimensional simple Lie algebra $\mathfrak{g}$ : Given nontrivial finite-dimensional irreducible $\mathfrak{g}$-modules $V_{1}, \ldots, V_{r}$ and $W_{1}, \ldots, W_{s}$ such that

$$
V_{1} \otimes \ldots \otimes V_{r} \cong W_{1} \otimes \ldots \otimes W_{s} \text { as } \mathfrak{g} \text {-modules }
$$

if and only if $r=s$ and the factors are pairwise isomorphic as $\mathfrak{g}$-modules up to a permutation of indices. This statement is equivalent to the family of irreducible finitedimensional representations having unique factorization property in the Grothendieck ring of finite dimensional representations of $\mathfrak{g}$. Rajan proved his result by an inductive analysis of the characters of tensor products, by fixing one of the variables, and passing to a suitable lower rank Lie algebra. One of his motivations was to show that given that $\operatorname{End}(V)=\operatorname{End}(W)$ for finite-dimensional irreducible modules $V, W$, using the natural isomorphism $\operatorname{End}(V) \cong V \otimes V^{*}$, one has that either $V \cong W$ or $V \cong W^{*}$. A more direct and simpler proof of Rajan's theorem is obtained by the second author and Viswanath in [12] and they also obtained a natural generalization of Rajan's theorem to KacMoody algebras setting. A natural category of representations to consider for Kac-Moody algebras is the category $\mathcal{O}^{\text {int }}$, whose objects are integrable $\mathfrak{g}$-modules in category $\mathcal{O}$, since both proofs of $[11,12]$ heavily use the Kac-Weyl character formula.

For a Kac-Moody algebra, the tensor product of two irreducible integrable representations can be irreducible when the Dynkin diagram is not connected. So the unique factorization property can not hold for Kac-Moody algebras which are not indecomposable. Even for the indecomposable case, the unique factorization of tensor products fails in general as there exist non-trivial one dimensional representations in $\mathcal{O}^{\text {int }}$ if $\mathfrak{g} \neq[\mathfrak{g}, \mathfrak{g}]$. However this is the only obstruction we have, i.e. uniqueness still holds up to twisting by one-dimensional representations for an indecomposable Kac-Moody algebra $\mathfrak{g}$. Note that the one dimensional representations are precisely the units of the Grothendieck ring of the category $\mathcal{O}^{\text {int }}$. Thus the unique factorization property still holds in indecomposable Kac-Moody case up to reordering and multiplying by units.

We can therefore naturally ask whether such a unique factorization of tensor products theorem holds for the irreducible integrable representations of Borcherds-Kac-Moody algebras (BKM algebras for short), as they also admit a character formula similar to the Kac-Weyl character formula. These algebras are defined using a (possibly infinite) Cartan matrix with relaxed conditions (see Section 2.1, (1)-(4)). Particular motivation was brought to these algebras as they include the monster Lie algebra, acted on by the monster group and used in the monstrous moonshine conjectures [3], and the fake monster Lie algebra. Imposing further that the Cartan matrix is finite, we exclude the Monster but allow many representational theoretic results such as the Harish-Chandra theorem [10], BGG resolution [8] and the Kazdan-Lusztig conjecture [9]. 
However, there are more obstacles for unique factorization in the case of BKM algebras (even for the indecomposable cases) since their building blocks involve Heisenberg algebras. For example let $\mathfrak{g}$ be the BKM algebra with only one imaginary simple root and no real simple roots and let $L(\lambda)$ be the irreducible integrable representation of $\mathfrak{g}$ corresponding to the dominant weight $\lambda$. Then it is easy to see that $L\left(\lambda_{1}\right) \otimes \cdots \otimes L\left(\lambda_{r}\right) \cong L\left(\mu_{1}\right) \otimes \cdots \otimes L\left(\mu_{r}\right)$ if and only if $\sum_{i=1}^{r} \lambda_{i}=\sum_{j=1}^{r} \mu_{j}$. So, one can not expect to get the unique factorization property for tensor products of irreducible integrable representations for general BKM algebras as in the case of Kac-Moody algebras, even up to one dimensional twists. It is not hard to produce more counter examples when the BKM algebras have real simple roots (see the Section 4.3).

It is therefore natural to study when two tensor products (not necessarily same number of components) of irreducible integrable representations of BKM algebras will be isomorphic to another. In this paper, we address this problem in full generality for Borcherds-Kac-Moody algebras whose Borcherds-Cartan matrix is finite. We also prove that we do get the unique factorization of tensor products under some assumption on the integrable irreducible representations of BKM algebras which we call special, see Section 2.5 for the definition.

Theorem. Let $\mathfrak{g}$ be an indecomposable BKM algebra and let $r, s \in \mathbb{N}$ and let $V_{i}, 1 \leq i \leq r$ and $W_{j}, 1 \leq j \leq s$ be special irreducible $\mathfrak{g}$-modules in category $\mathcal{O}^{\text {int }}$ such that

$$
V_{1} \otimes \cdots \otimes V_{r} \cong W_{1} \otimes \cdots \otimes W_{s}
$$

Then we have $r=s$ and there exists a permutation $\sigma$ on $\{1, \cdots, r\}$ and one-dimensional $\mathfrak{g}-$ modules $Z_{i}, 1 \leq i \leq r$ such that $V_{i}=W_{\sigma(i)} \otimes Z_{i}$.

This theorem is a corollary of Theorem 4.2.1 which describes when two tensor products of integrable irreducible $\mathfrak{g}$-modules (here $\mathfrak{g}$ need not be indecomposable) are isomorphic to each other. We remark that the assumption that all irreducible modules appearing in the tensor product being special can be relaxed in terms of their highest weights, indeed we need only a weaker assumption on their highest weights (see Corollary 4.2.2 and the Remark 4.2.3).

We now explain the main strategy of our proof. Let $\lambda_{1}, \ldots, \lambda_{r}, \mu_{1}, \ldots, \mu_{s}$ be dominant weights of $\mathfrak{g}$ such that $V_{i}=L\left(\lambda_{i}\right)$ and $W_{j}=L\left(\mu_{j}\right)$ for all $1 \leq i \leq r, 1 \leq j \leq s$. Since the category $\mathcal{O}^{\text {int }}$ is completely reducible for any BKM algebra $\mathfrak{g}$ (see [2], [6]), the isomorphism of the tensor products of $\mathfrak{g}$-modules becomes equivalent to the equality of the corresponding characters. So, taking formal characters on the both sides of tensor products one gets:

$$
\operatorname{ch} L\left(\lambda_{1}\right) \cdots \operatorname{ch} L\left(\lambda_{r}\right)=\operatorname{ch} L\left(\mu_{1}\right) \cdots \operatorname{ch} L\left(\mu_{s}\right)
$$

Now by canceling out the Weyl denominators, simplifying we translate our main problem to the equality of product of the normalized Weyl numerators, 


$$
U_{\lambda_{1}} \cdots U_{\lambda_{r}}=U_{\mu_{1}} \cdots U_{\mu_{s}}
$$

(see Section 2.4 for the precise definition of $U_{\lambda}$ ). We show that both sides of the Equation (1.2) can be further factorized to a product

$$
\prod_{i=1}^{r} \prod_{j=1}^{r_{i}} U_{j}^{\lambda_{i}}=\prod_{i=1}^{s} \prod_{j=1}^{s_{i}} U_{j}^{\mu_{i}}
$$

where the terms $U_{j}^{\lambda}$ are parametrized by the connected components of graphs associated to $\lambda$. We show that this factorization to such terms is unique. We prove this using the logarithm techniques developed in [12]. If the graphs of $\lambda_{1}, \ldots, \lambda_{r}, \mu_{1}, \ldots, \mu_{s}$ are connected, then we get $r=s$ and $r_{i}=s_{j}=1$ for $i, j=1, \ldots, r$ and we obtain the unique factorization of the characters and using this we obtain the unique factorization of the tensor products of special integrable irreducible $\mathfrak{g}-$ modules.

In fact, we prove this unique factorization result for more general expressions than the product of normalized Weyl numerators. We add a parameter $\chi$ to the normalized Weyl numerators where $\chi$ is a homomorphism on $W \times Q_{+}^{\text {im }}$, where $W$ is the Weyl group of $\mathfrak{g}$ and $Q_{+}^{\mathrm{im}}$ is the set of non-negative integer linear combination of imaginary simple roots (see Section 3.2 for more details). The unique factorization property is proved for any $\chi$. When $\chi$ is the sign character, we obtain the unique factorization properties of normalized Weyl numerators and hence the characters.

The paper is organized as follows: In Section 2, we set up the notations and preliminaries. In Section 3, we introduce the elements $U(\lambda, \chi)$ generalizing the normalized Weyl numerators associated with a homomorphism $\chi$ and prove the key properties concerning logarithm of $U(\lambda, \chi)$. In Section 4 , we use these properties to prove our main results of the paper.

\section{Acknowledgment}

The authors are thankful to Maria Gorelik for helpful conversations. This research was partially supported by the Minerva Foundation with funding from the Federal German Ministry for Education and Research.

\section{Preliminaries}

\subsection{Borcherds-Kac-Moody algebra}

Throughout this paper our base field will be complex numbers, i.e., all the algebras and representations are complex-vector spaces. The complex numbers, integers, non-negative integers, and positive integers are denoted by $\mathbb{C}, \mathbb{Z}, \mathbb{Z}_{+}$, and $\mathbb{N}$. We recall the definition of BKM algebras from [5]; they are also called generalized Kac-Moody algebras, see also 
[2,7]. A real matrix $A=\left(a_{i j}\right)_{i, j \in I}$ indexed by a finite set $I=\{1, \ldots, n\}$ is said to be a Borcherds-Cartan matrix if the following conditions are satisfied for all $i, j \in I$ :

(1) $A$ is symmetrizable;

(2) $a_{i i}=2$ or $a_{i i} \leq 0$;

(3) $a_{i j} \leq 0$ if $i \neq j$ and $a_{i j} \in \mathbb{Z}$ if $a_{i i}=2$;

(4) $a_{i j}=0$ if and only if $a_{j i}=0$.

Recall that a matrix $A$ is called symmetrizable if $D A$ is symmetric for some diagonal matrix $D=\operatorname{diag}\left(d_{1}, \ldots, d_{n}\right)$ with positive entries. Denote by $I^{\text {re }}=\left\{i \in I: a_{i i}=2\right\}$ and $I^{\mathrm{im}}=I \backslash I^{\mathrm{re}}$. The BKM algebra $\mathfrak{g}=\mathfrak{g}(A)$ associated to the Borcherds-Cartan matrix $A$ is the Lie algebra generated by $e_{i}, f_{i}, h_{i}, i \in I$ with the following defining relations:

(R1) $\left[h_{i}, h_{j}\right]=0$ for $i, j \in I$

(R2) $\left[h_{i}, e_{k}\right]=a_{i, k} e_{i},\left[h_{i}, f_{k}\right]=-a_{i, k} f_{i}$ for $i, k \in I$

(R3) $\left[e_{i}, f_{j}\right]=\delta_{i j} h_{i}$ for $i, j \in I$

(R4) $\left(\operatorname{ad} e_{i}\right)^{1-a_{i j}} e_{j}=0,\left(\operatorname{ad} f_{i}\right)^{1-a_{i j}} f_{j}=0$ if $i \in I^{\mathrm{re}}$ and $i \neq j$

(R5) $\left[e_{i}, e_{j}\right]=0$ and $\left[f_{i}, f_{j}\right]=0$ if $a_{i j}=0$.

Remark 2.1.1. If $i \in I$ is such that $a_{i i}=0$, the subalgebra spanned by the elements $h_{i}, e_{i}, f_{i}$ is isomorphic to the three dimensional Heisenberg algebra and otherwise it is isomorphic to $\mathfrak{s l}_{2}$. Note that we only consider the BKM algebras associated with finite Borcherds-Cartan matrices in this paper.

\subsection{Root system}

In this subsection, we recall some of the basic properties of BKM algebras; see [5] for more details. The BKM algebra $\mathfrak{g}$ is $\mathbb{Z}^{n}$-graded by defining

$$
\operatorname{deg} h_{i}=(0, \ldots, 0), \operatorname{deg} e_{i}=(0, \ldots, 0,1,0, \ldots, 0) \text { and } \operatorname{deg} f_{i}=(0, \ldots, 0,-1,0, \ldots, 0)
$$

where \pm 1 appears at the $i$-th position. For a sequence $\left(k_{1}, \ldots, k_{n}\right)$, we denote by $\mathfrak{g}\left(k_{1}, \ldots, k_{n}\right)$ the corresponding graded piece. Let $\mathfrak{h}=\operatorname{Span}_{\mathbb{C}}\left\{h_{i}: i \in I\right\}$ be the abelian subalgebra and let $\mathfrak{E}=\operatorname{Span}_{\mathbb{C}}\left\{D_{i}: i \in I\right\}$, where $D_{i}$ denotes the derivation that acts on $\mathfrak{g}\left(k_{1}, \ldots, k_{n}\right)$ by multiplication by the scalar $k_{i}$ and zero on the other graded components. Note that $D_{i}, i \in I$ are commuting derivations of $\mathfrak{g}$. The abelian subalgebra $\mathfrak{E} \ltimes \mathfrak{h}$ of $\mathfrak{E} \ltimes \mathfrak{g}$ acts by scalars on $\mathfrak{g}\left(k_{1}, \ldots, k_{n}\right)$ and giving a root space decomposition:

$$
\mathfrak{g}=\bigoplus_{\alpha \in(\mathfrak{E} \ltimes \mathfrak{h})^{*}} \mathfrak{g}_{\alpha}, \text { where } \mathfrak{g}_{\alpha}:=\{x \in \mathfrak{g} \mid[h, x]=\alpha(h) x \text { for all } h \in \mathfrak{E} \ltimes \mathfrak{h}\} .
$$

Define $\Pi=\left\{\alpha_{i}: i \in I\right\} \subseteq(\mathfrak{E} \ltimes \mathfrak{h})^{*}$ by $\alpha_{j}\left(\left(D_{k}, h_{i}\right)\right)=\delta_{k, j}+a_{i, j}$. The elements of $\Pi$ are called the simple roots of $\mathfrak{g}$. Set 


$$
Q:=\bigoplus_{\alpha \in \Pi} \mathbb{Z} \alpha, \quad Q_{+}:=\sum_{\alpha \in \Pi} \mathbb{Z}_{+} \alpha \text { and }
$$

The coroot associated with $\alpha \in \Pi$ is denoted by $h_{\alpha}$, namely $h_{\alpha}=h_{i}$ for $\alpha=\alpha_{i}$. The set of roots is denoted by $\Delta:=\left\{\alpha \in(\mathfrak{E} \ltimes \mathfrak{h})^{*} \backslash\{0\} \mid \mathfrak{g}_{\alpha} \neq 0\right\}$ and the set of positive roots is denoted by $\Delta_{+}:=\Delta \cap Q_{+}$. The elements in $\Pi^{\mathrm{re}}:=\left\{\alpha_{i}: i \in I^{\mathrm{re}}\right\}$ and $\Pi^{\mathrm{im}}:=\Pi \backslash \Pi^{\mathrm{re}}$ are called the set of real simple roots and the set of imaginary simple roots. Set $Q_{+}^{\mathrm{im}}:=\sum_{\alpha \in \Pi^{\mathrm{im}}} \mathbb{Z}_{+} \alpha$. We have $\Delta=\Delta_{+} \sqcup-\Delta_{+}$and

$$
\mathfrak{g}_{0}=\mathfrak{h}, \quad \mathfrak{g}_{\alpha}=\mathfrak{g}\left(k_{1}, \ldots, k_{n}\right), \quad \text { if } \quad \alpha=\sum_{i \in I} k_{i} \alpha_{i} \in \Delta .
$$

Moreover, we have a triangular decomposition $\mathfrak{g} \cong \mathfrak{n}^{-} \oplus \mathfrak{h} \oplus \mathfrak{n}^{+}$, where $\mathfrak{n}^{ \pm}=\bigoplus_{\alpha \in \pm \Delta_{+}} \mathfrak{g}_{\alpha}$. Given $\gamma=\sum_{i \in I} k_{i} \alpha_{i} \in Q_{+}$, we set $h t(\gamma)=\sum_{i \in I} k_{i}$. Finally, for $\lambda, \mu \in(\mathfrak{E} \ltimes \mathfrak{h})^{*}$ we say that $\lambda \geq \mu$ if $\lambda-\mu \in Q_{+}$.

\subsection{The Weyl group}

The real vector space spanned by $\Delta$ is denoted by $R=\mathbb{R} \otimes_{\mathbb{Z}} Q$. There is a symmetric bilinear form on $R$ given by $\left(\alpha_{i}, \alpha_{j}\right)=d_{i} a_{i j}$ for $i, j \in I$. For $\alpha \in \Pi^{\text {re }}$, define the linear isomorphism $\mathbf{s}_{\alpha}$ of $R$ by

$$
\mathbf{s}_{\alpha}(\lambda)=\lambda-2 \frac{(\lambda, \alpha)}{(\alpha, \alpha)} \alpha, \quad \lambda \in R
$$

The Weyl group $W$ of $\mathfrak{g}$ is the subgroup of $\mathrm{GL}(R)$ generated by the simple reflections $\mathbf{s}_{\alpha}, \alpha \in \Pi^{\mathrm{re}}$. Note that the above bilinear form is $W$-invariant and $W$ is a Coxeter group with canonical generators $\mathbf{s}_{\alpha}, \alpha \in \Pi^{\mathrm{re}}$. Define the length of $w \in W$ by $\ell(w):=\min \{k \in$ $\left.\mathbb{N}: w=\mathbf{s}_{\alpha_{i_{1}}} \cdots \mathbf{s}_{\alpha_{i_{k}}}\right\}$ and any expression $w=\mathbf{s}_{\alpha_{i_{1}}} \cdots \mathbf{s}_{\alpha_{i_{k}}}$ with $k=\ell(w)$ is called a reduced expression. The set of real roots is denoted by $\Delta^{\mathrm{re}}=W\left(\Pi^{\mathrm{re}}\right)$ and the set of imaginary roots is denoted by $\Delta^{\mathrm{im}}=\Delta \backslash \Delta^{\text {re }}$. Equivalently, a root $\alpha$ is real if and only if $(\alpha, \alpha)>0$ and else imaginary. We can extend (.,.) to a symmetric form on $(\mathfrak{E} \ltimes \mathfrak{h})^{*}$ satisfying $\left(\lambda, \alpha_{i}\right)=\lambda\left(d_{i} h_{i}\right)$ and also $\mathbf{s}_{\alpha}$ to a linear isomorphism of $(\mathfrak{E} \ltimes \mathfrak{h})^{*}$ by

$$
\mathbf{s}_{\alpha}(\lambda)=\lambda-2 \frac{(\lambda, \alpha)}{(\alpha, \alpha)} \alpha, \quad \lambda \in(\mathfrak{E} \ltimes \mathfrak{h})^{*} .
$$

Note that $\lambda\left(h_{\alpha}\right)=2 \frac{(\lambda, \alpha)}{(\alpha, \alpha)}$ for $\alpha \in \Pi$. Let $\rho$ be any element of $(\mathfrak{E} \ltimes \mathfrak{h})^{*}$ satisfying $2(\rho, \alpha)=(\alpha, \alpha)$ for all $\alpha \in \Pi$.

\subsection{Characters}

Denote by $\mathcal{O}^{\text {int }}$ the category consisting of integrable $\mathfrak{g}$-modules from the category $\mathcal{O}$ of $\mathfrak{g}$. Let $P_{+}=\left\{\lambda \in(\mathfrak{E} \ltimes \mathfrak{h})^{*}: \lambda\left(h_{\alpha}\right) \in \mathbb{Z}_{+}, \alpha \in \Pi\right\}$ be the set of all dominant integral weights of $\mathfrak{g}$ and let $L(\lambda)$ be the irreducible highest weight module of $\mathfrak{g}$ 
associated to $\lambda \in P_{+}$. Then it is well-known that there exists a bijective correspondence between the irreducible objects in $\mathcal{O}^{\text {int }}$ and $\left\{L(\lambda): \lambda \in P_{+}\right\}$and the category $\mathcal{O}^{\text {int }}$ is semi-simple (see $[2,6]$ ). Given $\lambda \in P_{+}$, the module $L(\lambda)$ has a weight space decomposition $L(\lambda)=\bigoplus_{\mu \in \mathfrak{h}^{*}} L(\lambda)_{\mu}$. The formal character of $L(\lambda)$ is defined to be $\operatorname{ch} L(\lambda):=\sum_{\mu \in \mathfrak{h}^{*}} \operatorname{dim}\left(L(\lambda)_{\mu}\right) e^{\mu}$. Let $\Omega(\lambda)$ be the set of all $\gamma \in Q_{+}$such that $\gamma$ is the sum of mutually orthogonal distinct imaginary simple roots which are orthogonal to $\lambda$. We define the normalized Weyl numerator by:

$$
U_{\lambda}:=\sum_{(w, \gamma) \in W \times \Omega(\lambda)}(-1)^{\ell(w)+\mathrm{ht}(\gamma)} e^{w(\lambda+\rho-\gamma)-(\lambda+\rho)} .
$$

Note that $0 \in \Omega(\lambda)$ and that an imaginary simple root $\alpha$ is in $\Omega(\lambda)$ if $(\lambda, \alpha)=0$. The Weyl-Kac character formula gives:

$$
\operatorname{ch} L(\lambda) e^{-\lambda}=\frac{\sum_{(w, \gamma) \in W \times \Omega(\lambda)}(-1)^{\ell(w)+h t(\gamma)} e^{w(\lambda+\rho-\gamma)-(\lambda+\rho)}}{\sum_{(w, \gamma) \in W \times \Omega(0)}(-1)^{\ell(w)+h t(\gamma)} e^{(w \rho-\rho-w \gamma)}}=\frac{U_{\lambda}}{U_{0}}
$$

Denote by $\mathcal{A}=\mathbb{C}\left[\left[X_{\alpha}: \alpha \in \Pi\right]\right]$ the formal power series ring on the variables $X_{\alpha}=e^{-\alpha}$ corresponding to the simple roots of $\mathfrak{g}$. Since $e^{\mu}$ appears in $\operatorname{ch} L(\lambda) e^{-\lambda}$ with nonzero coefficient only when $\lambda \geq \mu$, it follows that $U_{\lambda} \in \mathcal{A}$ for all $\lambda \in P_{+}$.

\subsection{Special dominant weights}

A dominant weight $\lambda \in P_{+}$is said to be special if $(\lambda, \alpha)=0$ for all $\alpha \in \Pi^{\mathrm{im}}$. An integrable irreducible $\mathfrak{g}$-module $L(\lambda)$ is called special if $\lambda$ is special. We record the following simple lemma for our future use.

Lemma 2.5.1. Let $\lambda \in P_{+}$. Then we have, $\lambda$ is special and $W$-invariant if and only if $L(\lambda)$ is one-dimensional if and only if $\operatorname{ch} L(\lambda)=e^{\lambda}$.

Note that, the irreducible $\mathfrak{g}$-module $L(\lambda)$ need not be one-dimensional in general for $\lambda \in P_{+}$which is $W$-invariant. Consider for example the case where all simple roots are isotropic, then the Weyl group is trivial. It is not hard to see that $\operatorname{ch} L(\lambda)=e^{\lambda}$ if and only if $\Omega(\lambda)=\Omega(0)$ if and only if $\lambda$ is special.

2.6. In this subsection, we recall some basic definitions and results about general simple graphs that were used to prove that unique factorization property in the KacMoody case (see [12, Section 4.2]). A graph $\mathcal{G}$ is said to be simple if it does not contain multiple edges (parallel edges) and loops.

Let $\mathcal{G}$ be a simple graph with the vertex set $V$ consisting of $n$ elements. For $S \subseteq V$, the subgraph spanned by $S$ is again a graph, denoted by $\mathcal{G}(S)$, whose vertex set is $S$ and there is an edge between the vertices $u, v \in S$ in $\mathcal{G}(S)$ if and only if there is an edge 
between the vertices $u, v \in S$ in $\mathcal{G}$. We say a subset $S$ of $V$ is independent if there is no edge between any two elements of $S$. The following definition is important for describing the factors of $U_{\lambda}$ (see also [12, Section 4.2]).

Definition 2.6.1. A $k$-partition of the graph $\mathcal{G}$ is an ordered $k$-tuple $\left(J_{1}, J_{2}, \ldots, J_{k}\right)$ such that the following conditions hold

(1) the $J_{i}$ are non-empty pairwise disjoint subsets of the vertex set $V$ whose union is $V$;

(2) each $J_{i}$ is an independent subset of $V$.

We denote by $P_{k}(\mathcal{G})$ the set of all $k$-partitions of $\mathcal{G}$ and $c_{k}(\mathcal{G}):=\left|P_{k}(\mathcal{G})\right|$. We also define

$$
c(\mathcal{G}):=(-1)^{n} \sum_{k=1}^{n}(-1)^{k} \frac{c_{k}(\mathcal{G})}{k} .
$$

The following result describes the connection between $c(G)$ and the connectivity.

Proposition 2.6.2. [12, Proposition 2] For a graph $\mathcal{G}$, we have $c(\mathcal{G}) \in \mathbb{Z}_{+}$and $c(\mathcal{G})>0$ if and only if $\mathcal{G}$ is connected.

\subsection{Graph of $\mathfrak{g}$ and $\lambda$}

Let $G$ be the simple graph underlying the Dynkin diagram of $\mathfrak{g}$, i.e. the vertex set of $G$ is $\Pi$ and two vertices $\alpha, \beta \in \Pi$ is connected by an edge if and only if $(\alpha, \beta) \neq 0$. We will refer to $G$ as the graph of $\mathfrak{g}$. For any subset $S \subseteq \Pi$, we denote $|S|$ by the number elements in $S$ and recall that $G(S)$ denotes the subgraph spanned by the subset $S$. We say a subset $S \subseteq \Pi$ is connected if the corresponding subgraph spanned by $S$ is connected, i.e., $G(S)$ is connected. For $\lambda \in P_{+}$, define

$$
\lambda_{\mathrm{im}}^{\perp}=\left\{\alpha \in \Pi^{\mathrm{im}}:(\lambda, \alpha)=0\right\} \text { and } \Pi(\lambda)=\Pi^{\mathrm{re}} \cup \lambda_{\mathrm{im}}^{\perp} .
$$

Note that the set $\Pi(\lambda)$ need not be connected in general and $\Pi(\lambda)=\Pi$ if $\lambda$ is special. The subgraph spanned by $\Pi(\lambda)$ will be called the graph of $\lambda$. Denote $\mathcal{C}(\lambda)$ by the set of all connected subsets of $\Pi(\lambda)$. The set of all connected components of $\Pi(\lambda)$ is denoted as $\mathcal{M C}(\lambda)$ and let $k_{\lambda}=|\mathcal{M C}(\lambda)|$ denote the number of connected components of $\Pi(\lambda)$. More generally for $\bar{\lambda}=\left(\lambda_{1}, \cdots, \lambda_{k}\right) \in P_{+}^{k}$, we denote $\mathcal{M C}(\bar{\lambda})$ to be the multiset $\sqcup_{i=1}^{k} \mathcal{M C}\left(\lambda_{i}\right)$. Finally, we denote by $S_{k}$ the set of permutations of $\{1, \cdots, k\}$ for $k \in \mathbb{N}$. 


\section{Logarithm expansion of characters}

In this section, we introduce the elements $U(\lambda, \chi)$ and $U_{i}(\lambda, \chi)$ that generalize the normalized Weyl numerator $U_{\lambda}$. We give all the arithmetic tools to prove criteria for their factorization, namely logarithm of characters and projection operators.

\subsection{The Weyl group action}

We discuss the Weyl group action on the elements $\lambda+\rho-\gamma, \lambda \in P_{+}$and $\gamma \in \Omega(\lambda)$ in order to understand the monomials $e^{w(\lambda+\rho-\gamma)-(\lambda+\rho)}$. For $w \in W$, we fix a reduced word expression $w=\mathbf{s}_{i_{1}} \cdots \mathbf{s}_{i_{k}}$ and let $I(w)=\left\{\alpha_{i_{1}}, \ldots, \alpha_{i_{k}}\right\} \subseteq \Pi^{\mathrm{re}}$. Note that $I(w)$ is independent of the choice of the reduced expression of $w$ (see [4]). Let $\lambda \in P_{+}$and for $\gamma \in \Omega(\lambda)$ we set

$$
I(\gamma)=\left\{\alpha \in \Pi^{\mathrm{im}}: \alpha \text { is a summand of } \gamma\right\}
$$

namely $I(\gamma)=\left\{\alpha_{i_{1}}, \cdots, \alpha_{i_{r}}\right\}$ if $\gamma=\sum_{k=1}^{r} \alpha_{i_{k}}$. Note that the set $I(\gamma)$ depends on $\lambda$, but we simply denote it as $I(\gamma)$ for convenience. Define

$$
\mathcal{J}(\lambda):=\{(w, \gamma) \in W \times \Omega(\lambda) \backslash\{(1,0)\}: I(w) \cup I(\gamma) \text { is an independent subset of } \Pi\} \text {. }
$$

Note that $(w, 0) \in \mathcal{J}(\lambda)$ if and only if $I(w)$ is an independent subset of $\Pi^{\text {re }}$ and $(1, \gamma) \in$ $\mathcal{J}(\lambda)$ for all nonzero $\gamma \in \Omega(\lambda)$. We record the following simple lemma for future use (see $[4])$.

Lemma 3.1.1. If $I(w)$ is an independent subset of $\Pi^{\mathrm{re}}$ for $w \in W$, then we have $\ell(w)=$ $|I(w)|$.

Given $\lambda \in P_{+}, w \in W$ and $\gamma \in \Omega(\lambda)$, we define $b_{\alpha}^{\lambda}(w, \gamma) \in \mathbb{Z}$ for every $\alpha \in \Pi$ by $(\lambda+\rho)-w(\lambda+\rho-\gamma)=\sum_{\alpha \in \Pi} b_{\alpha}^{\lambda}(w, \gamma) \alpha$.

Lemma 3.1.2. For a given $\lambda \in P_{+}, w \in W$ and $\gamma \in \Omega(\lambda)$, we have:

(i) $b_{\alpha}^{\lambda}(w, \gamma) \in \mathbb{Z}_{+}$for all $\alpha \in \Pi$ and $b_{\alpha}^{\lambda}(w, \gamma)=0$ if $\alpha \notin I(w) \cup I(\gamma)$;

(ii) $I(w)=\left\{\alpha \in \Pi^{\mathrm{re}}: b_{\alpha}^{\lambda}(w, \gamma) \geq \frac{2(\lambda+\rho, \alpha)}{(\alpha, \alpha)}\right\}$;

(iii) If $(w, \gamma) \in \mathcal{J}(\lambda)$, then $b_{\alpha}^{\lambda}(w, \gamma)=\frac{2(\lambda+\rho, \alpha)}{(\alpha, \alpha)}$ for all $\alpha \in I(w), b_{\alpha}^{\lambda}(w, \gamma)=1$ if $\alpha \in I(\gamma)$, and $b_{\alpha}^{\lambda}(w, \gamma)=0$, otherwise;

(iv) If $(w, \gamma) \notin \mathcal{J}(\lambda) \cup\{(1,0)\}$, then $\exists \alpha \in I(w) \subseteq \Pi^{\mathrm{re}}$ such that $b_{\alpha}^{\lambda}(w, \gamma)>\frac{2(\lambda+\rho, \alpha)}{(\alpha, \alpha)}$.

Proof. We prove the lemma by induction on $\ell(w)$. If $\ell(w)=0$, then (i)-(iii) are obvious. Let $\alpha \in \Pi^{\mathrm{re}}$ such that $w=\mathbf{s}_{\alpha} u$ and $\ell(w)=\ell(u)+1$. Then 


$$
\begin{aligned}
(\lambda+\rho)-w(\lambda+\rho-\gamma)= & (\lambda+\rho)-\mathbf{s}_{\alpha} u(\lambda+\rho-\gamma) \\
= & (\lambda+\rho)-u(\lambda+\rho-\gamma) \\
& +\left(2 \frac{\left(\lambda, u^{-1} \alpha\right)}{(\alpha, \alpha)}+2 \frac{\left(\rho, u^{-1} \alpha\right)}{(\alpha, \alpha)}-2 \frac{\left(\gamma, u^{-1} \alpha\right)}{(\alpha, \alpha)}\right) \alpha .
\end{aligned}
$$

By induction hypothesis we know that $(\lambda+\rho)-u(\lambda+\rho-\gamma)$ has the required property, and since $\ell(w)=\ell(u)+1$ and $\alpha \in \Pi^{\text {re }}$, we also know $u^{-1} \alpha \in \Delta^{\text {re }} \cap \Delta_{+}$. This implies that $2 \frac{\left(\lambda+\rho, u^{-1} \alpha\right)}{(\alpha, \alpha)} \in \mathbb{N}$, because $\lambda \in P_{+}$. Further, $\gamma$ is a sum of imaginary simple roots and $a_{i j} \leq 0$ whenever $i \neq j$. Hence $-2 \frac{\left(\gamma, u^{-1} \alpha\right)}{(\alpha, \alpha)} \in \mathbb{Z}_{+}$and since $I(w)=I(u) \cup\{\alpha\}$, the proof of (i) is done. To prove the Statement (ii), observe that there are two possibilities: $I(w)=I(u)$ or $I(w)=I(u) \sqcup\{\alpha\}$. Statement (ii) is immediate if $I(w)=I(u)$. Suppose $I(w)=I(u) \sqcup\{\alpha\}$, then we have $u^{-1} \alpha-\alpha \in Q_{+}$since $u^{-1} \alpha \in \Delta^{\text {re }} \cap \Delta_{+}$and $\alpha \notin I(u)$. This implies that $2 \frac{\left(\lambda+\rho, u^{-1} \alpha-\alpha\right)}{(\alpha, \alpha)} \geq 0$ as $\lambda \in P_{+}$. Hence (ii) follows.

If $(w, \gamma) \in \mathcal{J}(\lambda)$, then we have $I(w)=I(u) \sqcup\{\alpha\}$ and $(u, \gamma) \in \mathcal{J}(\lambda)$. This implies that $u^{-1} \alpha=\alpha$ and so $\left(\rho, u^{-1} \alpha\right)=(\rho, \alpha)=\frac{1}{2}(\alpha, \alpha)$ and $\left(\gamma, u^{-1} \alpha\right)=(\gamma, \alpha)=0$. Thus (iii) follows from the above expression of $(\lambda+\rho)-w(\lambda+\rho-\gamma)$ by induction.

It remains to prove (iv). Suppose $w=\mathbf{s}_{\alpha}$ we have

$$
(\lambda+\rho)-w(\lambda+\rho-\gamma)=\gamma+\left(1+2 \frac{(\lambda, \alpha)}{(\alpha, \alpha)}-2 \frac{(\gamma, \alpha)}{(\alpha, \alpha)}\right) \alpha
$$

Since $(w, \gamma) \notin \mathcal{J}(\lambda) \cup\{(1,0)\},\{\alpha\}$ and $I(\gamma)$ are not independent and we get $-2 \frac{(\gamma, \alpha)}{(\alpha, \alpha)} \in \mathbb{N}$. This completes the proof of (iv) for $w=\mathbf{s}_{\alpha}$, giving us a base for induction. For the induction step we write $w=\mathbf{s}_{\alpha} u$ where $\ell(w)=\ell(u)+1$. We have either $(u, \gamma) \in$ $\mathcal{J}(\lambda) \cup\{(1,0)\}$ or $(u, \gamma) \notin \mathcal{J}(\lambda) \cup\{(1,0)\}$. In the latter case, we are done by using the induction hypothesis since $I(u) \subseteq I(w)$. So, assume that $(u, \gamma) \in \mathcal{J}(\lambda) \cup\{(1,0)\}$. Since $(w, \gamma) \notin \mathcal{J}(\lambda) \cup\{(1,0)\}$, we have $(u, \gamma) \neq(1,0)$ and $I(w)=I(u) \sqcup\{\alpha\}$ and there are two possibilities: either $I(u) \cup\{\alpha\}$ is independent or $\{\alpha\} \cup I(\gamma)$ is independent. However we can not have that both of them are independent.

Case 1: Suppose $I(u) \cup\{\alpha\}$ is independent and $\{\alpha\} \cup I(\gamma)$ is not independent, then we have $\left(\gamma, u^{-1} \alpha\right)=(\gamma, \alpha)<0$ and $-2 \frac{\left(\gamma, u^{-1} \alpha\right)}{(\alpha, \alpha)} \in \mathbb{N}$, so we are done by interpreting this in the above expression of $(\lambda+\rho)-w(\lambda+\rho-\gamma)$.

Case 2. Suppose $\{\alpha\} \cup I(\gamma)$ is independent and $I(u) \cup\{\alpha\}$ is not independent, then we have $u^{-1}(\alpha)-\alpha \in Q_{+} \backslash\{0\}$ since $u^{-1} \alpha \in \Delta^{\text {re }} \cap \Delta_{+}$and $\alpha \notin I(u)$. So, we get $2 \frac{\left(\lambda+\rho, u^{-1} \alpha-\alpha\right)}{(\alpha, \alpha)} \geq 1$. Now interpreting this in the above expression of $(\lambda+\rho)-w(\lambda+\rho-\gamma)$, the assertion follows.

Remark 3.1.3. The above lemma is a generalization of [12, Lemma 2] in the setting of BKM algebras, see also [1, Lemma 3.6] for the special case $\lambda=0$. 


\subsection{The elements $U(\lambda, \chi)$}

We define the elements $U(\lambda, \chi)$ in $\mathcal{A}$ which generalize the Weyl numerators $U_{\lambda}$. We shall later determine in which cases these elements admit unique factorization and how they factorize in other cases.

Let $\chi: W \times Q_{+}^{\mathrm{im}} \rightarrow \mathbb{C} \backslash\{0\}$ be a homomorphism that is,

$$
\chi(1,0)=1 \text { and } \chi\left(\prod_{i=1}^{r} w_{i}, \sum_{i=1}^{r} \gamma_{i}\right)=\chi\left(w_{1}, \gamma_{1}\right) \cdots \chi\left(w_{r}, \gamma_{r}\right)
$$

for $w_{1}, \ldots, w_{r} \in W$ and $\gamma_{1}, \ldots, \gamma_{r} \in Q_{+}^{\text {im }}$. For $\lambda \in P_{+}$and such $\chi$, we define the normalized Weyl numerator associated with $(\lambda, \chi)$ as follows:

$$
U(\lambda, \chi):=\sum_{(w, \gamma) \in W \times \Omega(\lambda)} \chi(w, \gamma) e^{w(\lambda+\rho-\gamma)-(\lambda+\rho)}
$$

Since $w(\lambda+\rho) \leq \lambda+\rho$ for all $w \in W$, we have $U(\lambda, \chi) \in \mathcal{A}$. There are two important homomorphisms which are especially useful. One is the trivial homomorphism defined to be

$$
\mathbb{1}(w, \gamma):=1
$$

for all $(w, \gamma) \in W \times Q_{+}^{\mathrm{im}}$ and another one is the sign homomorphism which is defined by

$$
\operatorname{sgn}(w, \gamma):=(-1)^{\ell(w)}(-1)^{\mathrm{ht}(\gamma)}
$$

Clearly, $U(\lambda, \operatorname{sgn})=U_{\lambda}$, that is $U(\lambda, \chi)$ generalizes the Weyl numerator, and

$$
U(\lambda, \mathbb{1})=\sum_{(w, \gamma) \in W \times \Omega(\lambda)} e^{w(\lambda+\rho-\gamma)-(\lambda+\rho)} .
$$

\subsection{Logarithm of $U(\lambda, \chi)$}

We recall the notion of logarithm of elements in $\mathcal{A}$ which is our main tool to make the product $U\left(\lambda_{1}, \chi\right) \cdots U\left(\lambda_{r}, \chi\right)$ into a sum $\sum_{i=1}^{r} \log U\left(\lambda_{i}, \chi\right)$ which is less difficult to analyze. Given an element $f \in \mathcal{A}$, we define

$$
-\log (1-f)=f+\frac{f^{2}}{2}+\frac{f^{3}}{3}+\cdots+\frac{f^{k}}{k}+\cdots
$$

Note that $-\log (1-f) \in \mathcal{A}$ for all $f \in \mathcal{A}$ and $\log (f g)=\log (f)+\log (g)$ for all elements $f, g \in \mathcal{A}$ with constant terms 1 . 
Now write $\zeta(\lambda, \chi):=1-U(\lambda, \chi)$ and for $(w, \gamma) \in W \times \Omega(\lambda)$ we denote the monomial

$$
X(\lambda, w, \gamma):=e^{w(\lambda+\rho-\gamma)-(\lambda+\rho)}=\prod_{\alpha \in \Pi} X_{\alpha}^{b_{\alpha}^{\lambda}(w, \gamma)} .
$$

For a subset $C$ of $\Pi$, denote by

$$
X^{\lambda}(C):=\prod_{\alpha \in C^{\mathrm{re}}} X_{\alpha}^{(\lambda+\rho)\left(h_{\alpha}\right)} \prod_{\alpha \in C^{\mathrm{im}}} X_{\alpha}
$$

and

$$
\chi(C)=\prod_{i \in C^{\mathrm{re}}} \chi\left(\mathbf{s}_{i}, 0\right) \prod_{i \in C^{\mathrm{im}}} \chi\left(1, \alpha_{i}\right)
$$

where $C^{\mathrm{re}}=\Pi^{\mathrm{re}} \cap C$ and $C^{\mathrm{im}}=\Pi^{\mathrm{im}} \cap C$.

One of the key ingredients in the proof of our main theorem is to we investigate the elements $-\log U(\lambda, \chi)$ and give necessary and sufficient conditions for the monomials $X^{\lambda}(C)$ to appear in $-\log U(\lambda, \chi)$ with nonzero coefficient. The following proposition is a generalization of [12, Proposition 1] for BKM algebras. Recall the definitions of $\Pi(\lambda)=\Pi^{\mathrm{re}} \cup \lambda_{\mathrm{im}}^{\perp}$ and $c(\mathcal{G})$ from the Sections 2.7 and 2.6.

Proposition 3.3.1. Let $\chi: W \times Q_{+}^{\mathrm{im}} \rightarrow \mathbb{C} \backslash\{0\}$ be a homomorphism and let $\lambda \in P_{+}$and $C \subseteq \Pi$.

(i) Suppose the monomial $\prod_{\alpha \in \Pi} X_{\alpha}^{a_{\alpha}}$ appears in $-\log U(\lambda, \chi)$ with nonzero coefficient then the support of this monomial must be contained in $\Pi(\lambda)$, i.e.

$$
\operatorname{supp}\left(\prod_{\alpha \in \Pi} X_{\alpha}^{a_{\alpha}}\right)=\left\{\alpha \in \Pi: a_{\alpha} \neq 0\right\} \subseteq \Pi(\lambda) .
$$

(ii) The coefficient of the monomial $X^{\lambda}(C)$ in $-\log U(\lambda, \chi)$ is equal to $\chi(C) c(G(C))$ for $C \subseteq \Pi(\lambda)$, where $G(C)$ is the subgraph spanned by $C$. In particular, this coefficient depends only on the subset $C$.

(iii) The monomial $X^{\lambda}(C)$ appears in $-\log U(\lambda, \chi)$ with nonzero coefficient if and only if $C$ is a connected subset of $\Pi(\lambda)$.

Proof. Part (iii) of the proposition follows from (ii) and the Proposition 2.6.2, since $\chi(C) \neq 0$ for all $C \subseteq \Pi$. Now write

$$
\zeta(\lambda, \chi)=-\sum_{\substack{(w, \gamma) \in W \times \Omega(\lambda) \\(w, \gamma) \neq(1,0)}} \chi(w, \gamma) X(\lambda, w, \gamma)=\zeta_{1}(\lambda, \chi)+\zeta_{2}(\lambda, \chi)
$$

where 


$$
\zeta_{1}(\lambda, \chi)=-\sum_{\substack{(w, \gamma) \in W \times \Omega(\lambda) \\(w, \gamma) \in \mathcal{J}(\lambda)}} \chi(w, \gamma) X(\lambda, w, \gamma)
$$

and

$$
\zeta_{2}(\lambda, \chi)=-\sum_{\substack{(w, \gamma) \in W \times \Omega(\lambda) \\(w, \gamma) \notin \mathcal{J}(\lambda) \cup\{(1,0)\}}} \chi(w, \gamma) X(\lambda, w, \gamma)
$$

Since $-\log U(\lambda, \chi)=\zeta(\lambda, \chi)+\frac{\zeta(\lambda, \chi)^{2}}{2}+\cdots+\frac{\zeta(\lambda, \chi)^{k}}{k}+\cdots$, Lemma 3.1.2 implies that any monomial $\prod_{\alpha \in \Pi^{\mathrm{re}}} X_{\alpha}^{p_{\alpha}} \prod_{\alpha \in \Pi^{\mathrm{im}}} X_{\alpha}^{m_{\alpha}}$ that occur in $-\log U(\lambda, \chi)$ with nonzero coefficient must satisfy the following conditions:

(1) if $p_{\alpha} \neq 0$ for some $\alpha \in \Pi^{\text {re }}$ then $p_{\alpha} \geq(\lambda+\rho)\left(h_{\alpha}\right)$ and $(2) m_{\alpha}=0$ for $\alpha \notin \Pi(\lambda)$.

This proves the statement (i) of the proposition. In particular, the monomial $X^{\lambda}(C)$ appears in $-\log U(\lambda, \chi)$ with nonzero coefficient then $C \subseteq \Pi(\lambda)$. Let $C \subseteq \Pi(\lambda)$, then Lemma 3.1.2 (iv) further implies that there is no contribution of $\zeta_{2}(\lambda, \chi)$ to the coefficient of the monomial $X^{\lambda}(C)$ in $\zeta(\lambda, \chi)+\frac{\zeta(\lambda, \chi)^{2}}{2}+\cdots+\frac{\zeta(\lambda, \chi)^{k}}{k}+\cdots$, i.e.

$$
\begin{aligned}
& \text { the coefficient of } X^{\lambda}(C) \text { in }\left(\sum_{k=1}^{\infty} \frac{\zeta(\lambda, \chi)^{k}}{k}\right) \\
& =\text { the coefficient of } X^{\lambda}(C) \text { in }\left(\sum_{k=1}^{\infty} \frac{\zeta_{1}(\lambda, \chi)^{k}}{k}\right) \\
& =\sum_{k=1}^{\infty}\left(\text { the coefficient of } X^{\lambda}(C) \text { in } \frac{\zeta_{1}(\lambda, \chi)^{k}}{k}\right) .
\end{aligned}
$$

Hence it is enough to calculate the coefficient of $X^{\lambda}(C)$ in $\zeta_{1}(\lambda, \chi)^{k}$, where

$$
\zeta_{1}(\lambda, \chi)^{k}=\sum_{\substack{\left(\left(w_{1}, \gamma_{1}\right),\left(w_{2}, \gamma_{2}\right), \ldots,\left(w_{k}, \gamma_{k}\right)\right) \\\left(w_{i}, \gamma_{i}\right) \in W \times \Omega(\lambda) \\\left(w_{i}, \gamma_{i}\right) \in \mathcal{J}(\lambda)}}(-1)^{k} \prod_{i=1}^{k} \chi\left(w_{i}, \gamma_{i}\right) X\left(\lambda, w_{i}, \gamma_{i}\right)
$$

From Lemma 3.1.2 (iii), we get that $\prod_{i=1}^{k} X\left(\lambda, w_{i}, \gamma_{i}\right)=X^{\lambda}(C)$ if and only if $\left(I\left(w_{1}\right) \cup\right.$ $\left.I\left(\gamma_{1}\right), I\left(w_{2}\right) \cup I\left(\gamma_{2}\right), \ldots, I\left(w_{k}\right) \cup I\left(\gamma_{k}\right)\right)$ form a $k$-partition of $C$. In particular, for this $k$-partition of $C$ we have

$$
\prod_{i=1}^{k} \chi\left(w_{i}, \gamma_{i}\right)=\chi\left(\prod_{i=1}^{k} w_{i}, \sum_{i=1}^{k} \gamma_{i}\right)=\prod_{i \in C^{\mathrm{re}}} \chi\left(\mathbf{s}_{i}, 0\right) \prod_{i \in C^{\mathrm{im}}} \chi\left(1, \alpha_{i}\right)=\chi(C)
$$


which is independent of the choice of the partition and depends only upon the subset $C$ and the homomorphism $\chi$. Since all the $k$-partitions of $C$ arise in this way and $\chi$ is a homomorphism, using Lemma 3.1.1, we have

$$
\text { (the coefficient of } \left.X^{\lambda}(C) \text { in } \zeta_{1}(\lambda, \gamma)^{k}\right)=(-1)^{k} \chi(C)\left|P_{k}(G(C))\right|
$$

where $G(C)$ is the subgraph spanned by $C$. Putting this all together, we conclude that (the coefficient of $X^{\lambda}(C)$ in $\left.-\log U(\lambda, \chi)\right)=\sum_{k=1}^{\infty} \frac{(-1)^{k}}{k} \chi(C) \mid P_{k}(G(C) \mid=\chi(C) c(G(C))$. Since $\chi(C) \neq 0$ for all $C \subseteq \Pi$, we have $\chi(C) c(G(C))$ is nonzero if and only if $c(G(C))$ is nonzero. Thus Proposition 2.6.2 completes the proof of (ii).

\subsection{Factorization of $U(\lambda, \chi)$}

The set $\Pi(\lambda)$ need not be connected in general for $\lambda \in P_{+}$. We factor $U(\lambda, \chi)$ to a product of elements $U_{i}(\lambda, \chi)$ which correspond to the connected components of the graph corresponding to $\Pi(\lambda)$.

Let $\lambda \in P_{+}$and let $C_{i}^{\lambda}, 1 \leq i \leq k_{\lambda}$, be the connected components of $\Pi(\lambda)$. For $1 \leq i \leq k_{\lambda}$, we denote by $W(\lambda)_{i}$ the Weyl group generated by the simple reflections $\left\{\mathbf{s}_{\alpha}: \alpha \in C_{i}^{\lambda} \cap \Pi^{\mathrm{re}}\right\}$. Similarly, for $1 \leq i \leq k_{\lambda}$, we denote by $\Omega(\lambda)_{i}$ the set of all $\gamma \in Q_{+}$ such that $\gamma$ is the sum of mutually orthogonal distinct imaginary simple roots which are in $C_{i}^{\lambda} \cap \lambda_{\mathrm{im}}^{\perp}$. Then we have

$$
U(\lambda, \chi)=\prod_{i=1}^{k_{\lambda}}\left(\sum_{(w, \gamma) \in W(\lambda)_{i} \times \Omega(\lambda)_{i}} \chi(w, \gamma) e^{w(\lambda+\rho-\gamma)-(\lambda+\rho)}\right)
$$

For $1 \leq i \leq k_{\lambda}$, we denote

$$
U_{i}(\lambda, \chi):=\sum_{(w, \gamma) \in W(\lambda)_{i} \times \Omega(\lambda)_{i}} \chi(w, \gamma) e^{w(\lambda+\rho-\gamma)-(\lambda+\rho)}
$$

Then Equation (3.1) becomes

$$
U(\lambda, \chi)=U_{1}(\lambda, \chi) \cdots U_{k_{\lambda}}(\lambda, \chi)
$$

In the following proposition, we make some observations about the monomials which appear in $-\log U_{i}(\lambda, \chi)$, which are similar to the observations about $U(\lambda, \chi)$ that were stated in Proposition 3.3.1.

Proposition 3.4.1. Let $\chi: W \times Q_{+}^{\mathrm{im}} \rightarrow \mathbb{C} \backslash\{0\}$ be a homomorphism and let $\lambda \in P_{+}$. Let $C_{1}^{\lambda}, \cdots, C_{k_{\lambda}}^{\lambda}$ be the connected components of $\Pi(\lambda)$. For $1 \leq i \leq k_{\lambda}$, we have: 
(i) The support of a monomial which appears in $-\log U_{i}(\lambda, \chi)$ with nonzero coefficient must be contained in $C_{i}^{\lambda}$.

(ii) The coefficient of the monomial $X^{\lambda}(C)$ in $-\log U_{i}(\lambda, \chi)$ is equal to $\chi(C) c(G(C))$ for any $C \subseteq C_{i}^{\lambda}$, and in particular this coefficient depends only on the subset $C$.

(iii) For a subset $C \subseteq C_{i}^{\lambda}$, the monomial $X^{\lambda}(C)$ appears in $-\log U_{i}(\lambda, \chi)$ with nonzero coefficient if and only if $C$ is a connected subset of $C_{i}^{\lambda}$.

Proof. We leave out the proof since it closely follows the arguments of Proposition 3.3.1.

\subsection{The projection operator $\#_{C}$}

We define the projection operator $\#_{C}$ corresponding to a connected component $C$ of the graph of $\lambda$. We apply this operator on $-\log U_{i}(\lambda, \chi)$ and use it to determine when $U_{i}(\lambda, \chi)$ is equal to $U_{j}(\mu, \chi)$.

Recall that $\mathcal{A}=\mathbb{C}\left[\left[X_{\alpha}: \alpha \in \Pi\right]\right]$ where $X_{\alpha}=e^{-\alpha}$. Let $\lambda \in P_{+}$and $C$ be a connected component of $\Pi(\lambda)$. Define the map $\#_{C}: \mathcal{A} \rightarrow \mathcal{A}$ which maps

$$
f=\sum_{\mathbf{a}} X_{\alpha}^{a_{\alpha}} \mapsto f^{\#_{C}}=\sum_{\substack{\mathbf{a} \\ \operatorname{supp}(\mathbf{a})=C}} X_{\alpha}^{a_{\alpha}}
$$

where $\mathbf{a}=\left(a_{\alpha}: \alpha \in \Pi\right)$ and $\operatorname{supp}(\mathbf{a})=\left\{\alpha \in \Pi: a_{\alpha} \neq 0\right\}$. It is easy to see that the operator $\#_{C}$ is a linear operator. The following proposition follows from Proposition 3.4.1 (which is based on Lemma 3.1.2(iv)). It plays a crucial role in the proof of our main theorem.

Proposition 3.5.1. Let $\chi: W \times Q_{+}^{\mathrm{im}} \rightarrow \mathbb{C} \backslash\{0\}$ be a homomorphism. Let $\lambda \in P_{+}$and $C=C_{i}^{\lambda}$ be a connected component of $\Pi(\lambda)$. Then we have

$$
\left(-\log U_{i}(\lambda, \chi)\right)^{\#_{C}}=\chi(C) c(G(C)) X^{\lambda}(C)+\text { monomials of degree }>\operatorname{deg} X^{\lambda}(C)
$$

where $\operatorname{deg} X^{\lambda}(C)=\sum_{\alpha \in C^{\mathrm{re}}} \lambda\left(h_{\alpha}\right)+\left|C^{\mathrm{im}}\right|$.

The following lemma compares when two $U_{i}(\lambda, \chi), U_{j}(\mu, \chi)$ are equal.

Lemma 3.5.2. Let $\chi: W \times Q_{+}^{\mathrm{im}} \rightarrow \mathbb{C} \backslash\{0\}$ be a homomorphism. Let $\lambda, \mu \in P_{+}$and let $C_{i}^{\lambda}$ and $C_{j}^{\mu}$ be two connected components $\Pi(\lambda)$ and $\Pi(\mu)$ respectively. Then the following statements are equivalent:

(i) $X^{\lambda}\left(C_{i}^{\lambda}\right)=X^{\mu}\left(C_{j}^{\mu}\right)$

(ii) $C_{i}^{\lambda}=C_{j}^{\mu}$ and $\lambda\left(h_{\alpha}\right)=\mu\left(h_{\alpha}\right)$ for all $\alpha \in C_{i}^{\lambda} \cap \Pi^{\mathrm{re}}$

(iii) $U_{i}(\lambda, \chi)=U_{j}(\mu, \chi)$ 
Proof. If $X^{\lambda}\left(C_{i}^{\lambda}\right)=X^{\mu}\left(C_{j}^{\mu}\right)$ then the supports and exponents of the corresponding variables of these monomials must be equal, so (ii) follows from (i). Assume (ii), we get $C_{i}^{\lambda} \cap \Pi^{\mathrm{re}}=C_{j}^{\mu} \cap \Pi^{\mathrm{re}}$ and $C_{i}^{\lambda} \cap \Pi^{\mathrm{im}}=C_{j}^{\mu} \cap \Pi^{\mathrm{im}}$. This immediately implies $W(\lambda)_{i}=$ $W(\mu)_{j}$, and $\Omega(\lambda)_{i}=\Omega(\mu)_{j}$. Since $\lambda\left(h_{\alpha}\right)=\mu\left(h_{\alpha}\right)$ for all $\alpha \in C_{i}^{\lambda} \cap \Pi^{\text {re }}$, we have $w(\lambda+$ $\rho)-(\lambda+\rho)=w(\mu+\rho)-(\mu+\rho)$ for all $w \in W(\lambda)_{i}$. Hence we have $U_{i}(\lambda, \chi)=U_{j}(\lambda, \chi)$. Finally, the fact that (iii) implies (i) follows from Proposition 3.5.1.

\section{Unique factorization properties}

4.1. In this section, we prove that the product $U\left(\lambda_{1}, \chi\right) \cdots U\left(\lambda_{r}, \chi\right)$ factorizes uniquely to terms corresponding to the connected components of $\Pi\left(\lambda_{1}\right), \ldots, \Pi\left(\lambda_{r}\right)$. More precisely, let $r, s \in \mathbb{N}$ and $\bar{\lambda}=\left(\lambda_{1}, \ldots, \lambda_{r}\right) \in P_{+}^{r}, \bar{\mu}=\left(\mu_{1}, \ldots, \mu_{s}\right) \in P_{+}^{s}$ such that

$$
U\left(\lambda_{1}, \chi\right) \cdots U\left(\lambda_{r}, \chi\right)=U\left(\mu_{1}, \chi\right) \cdots U\left(\mu_{s}, \chi\right)
$$

Recall that, we can write $U\left(\lambda_{p}, \chi\right)=\prod_{i=1}^{k_{\lambda_{p}}} U_{i}\left(\lambda_{p}, \chi\right)$ for $1 \leq p \leq r$ (see Equation (3.2)) and similarly, $U\left(\mu_{q}, \chi\right)=\prod_{j=1}^{k_{\mu_{q}}} U_{j}\left(\mu_{q}, \chi\right)$ for $1 \leq q \leq s$. Then Equation (4.1) becomes

$$
\prod_{p=1}^{r}\left(\prod_{i=1}^{k_{\lambda_{p}}} U_{i}\left(\lambda_{p}, \chi\right)\right)=\prod_{q=1}^{s}\left(\prod_{j=1}^{k_{\mu_{q}}} U_{j}\left(\mu_{q}, \chi\right)\right)
$$

We now prove that the factors occur in the Equation (4.2) are unique up to permutation. i.e., number of factors on the both sides are equal $\left(N=\sum_{p=1}^{r} k_{\lambda_{p}}=\sum_{q=1}^{s} k_{\mu_{q}}\right)$ and there exists a permutation $\sigma$ on $S_{N}$ such that $U_{i}\left(\lambda_{p}, \chi\right)=U_{i^{\prime}}\left(\mu_{p^{\prime}}, \chi\right)$ where $\sigma(i, p)=\left(i^{\prime}, p^{\prime}\right)$. Precisely,

Theorem 4.1.1. Let $\chi: W \times Q_{+}^{\mathrm{im}} \rightarrow \mathbb{C} \backslash\{0\}$ be a homomorphism. Let $r, s \in \mathbb{N}$ and let $\bar{\lambda}=\left(\lambda_{1}, \ldots, \lambda_{r}\right) \in P_{+}^{r}, \bar{\mu}=\left(\mu_{1}, \ldots, \mu_{s}\right) \in P_{+}^{s}$. Then the following statements are equivalent:

(1) $U\left(\lambda_{1}, \chi\right) \cdots U\left(\lambda_{r}, \chi\right)=U\left(\mu_{1}, \chi\right) \cdots U\left(\mu_{s}, \chi\right)$

(2) there exists a bijection $\sigma: \mathcal{M C}(\bar{\lambda}) \rightarrow \mathcal{M C}(\bar{\mu})$ given by $C \mapsto C_{\sigma}$ satisfying the following conditions: if $C \in \mathcal{M C}\left(\lambda_{i}\right)$ maps to $C_{\sigma} \in \mathcal{M C}\left(\mu_{j}\right)$ then

(i) $C=C_{\sigma}$ and further, (ii) $\lambda_{i}\left(h_{\alpha}\right)=\mu_{j}\left(h_{\alpha}\right)$ for all $\alpha \in C^{\text {re }}$.

(3) $\sum_{p=1}^{r} k_{\lambda_{p}}=\sum_{q=1}^{s} k_{\mu_{q}}=: N$ and there exists a permutation $\sigma \in S_{N}$ such that

$$
U_{i}\left(\lambda_{p}, \chi\right)=U_{i^{\prime}}\left(\mu_{p^{\prime}}, \chi\right) \text { where } \sigma(i, p)=\left(i^{\prime}, p^{\prime}\right)
$$


Proof. $(2) \Longleftrightarrow(3)$ follows from the Lemma 3.5.2 and $(3) \Longrightarrow(1)$ is obvious. Now we prove $(1) \Longrightarrow(3)$. Suppose

$$
U\left(\lambda_{1}, \chi\right) \cdots U\left(\lambda_{r}, \chi\right)=U\left(\mu_{1}, \chi\right) \cdots U\left(\mu_{s}, \chi\right)
$$

Factorize this further in terms of $U_{i}(\lambda, \chi)$ as before, then we get

$$
\prod_{p=1}^{r}\left(\prod_{i=1}^{k_{\lambda_{p}}} U_{i}\left(\lambda_{p}, \chi\right)\right)=\prod_{q=1}^{s}\left(\prod_{j=1}^{k_{\mu_{q}}} U_{j}\left(\mu_{q}, \chi\right)\right)
$$

Applying - $\log$ on both sides we get

$$
\sum_{p=1}^{r} \sum_{i=1}^{k_{\lambda_{p}}}-\log U_{i}\left(\lambda_{p}, \chi\right)=\sum_{q=1}^{s} \sum_{j=1}^{k_{\mu_{q}}}-\log U_{j}\left(\mu_{q}, \chi\right)
$$

Let $\mathcal{C}=\left\{C_{i}^{\lambda_{p}}: p \in\{1, \cdots, r\}, 1 \leq i \leq k_{\lambda_{p}}\right\} \cup\left\{C_{j}^{\mu_{q}}: q \in\{1, \cdots, s\}, 1 \leq j \leq k_{\mu_{q}}\right\}$ to be the set of all connected components of $\Pi\left(\lambda_{i}\right), \Pi\left(\mu_{j}\right), 1 \leq i \leq k_{\lambda_{p}}, 1 \leq j \leq k_{\mu_{q}}$. We fix a maximal element $C$ from $\mathcal{C}$ with respect to set inclusion. Without loss of generality we assume that this chosen maximal element occurs on the left hand side of the Equation (4.5), say $C=C_{i_{0}}^{\lambda_{1}}$, and satisfies the following property: the monomial $X^{\lambda}(C)$ is the minimal degree monomial (with respect to total degree) among all the monomials in the left hand side of the Equation (4.5) with $C$ as support, where $\lambda_{1}=\lambda$. Now apply the map $\#_{C}$ to Equation (4.5) then we get

$$
\sum_{p=1}^{r} \sum_{i=1}^{k_{\lambda_{p}}}-\log U_{i}\left(\lambda_{p}, \chi\right)^{\#_{C}}=\sum_{q=1}^{s} \sum_{j=1}^{k_{\mu_{q}}}-\log U_{j}\left(\mu_{q}, \chi\right)^{\#_{C}} .
$$

Since $C$ is maximal in $\mathcal{C}$ with respect to set inclusion, we immediately observe the following from Proposition 3.4.1:

(i) either $-\log U_{i}\left(\lambda_{p}, \chi\right)^{\#_{C}}$ is equal to zero or

(ii) we have $C_{i}^{\lambda_{p}}=C$ and the monomial $X^{\lambda_{p}}(C)$ is the minimal degree monomial in $-\log U_{i}\left(\lambda_{p}, \chi\right)^{\#_{C}}$ with respect to the total degree when $-\log U_{i}\left(\lambda_{p}, \chi\right)^{\#_{C}}$ is nonzero.

Similarly we have:

(iii) either $-\log U_{j}\left(\mu_{q}, \chi\right)^{\#_{C}}$ is equal to zero or

(iv) we have $C_{j}^{\mu_{q}}=C$ and the monomial $X^{\mu_{q}}(C)$ is the minimal degree monomial in $-\log U_{j}\left(\mu_{q}, \chi\right)^{\#_{C}}$ with respect to the total degree when $-\log U_{j}\left(\mu_{q}, \chi\right)^{\#_{C}}$ is nonzero. 
Since $X^{\lambda}(C)$ appears in the left hand side of Equation (4.6) with nonzero coefficient and it is of minimal degree monomial, there must exist $1 \leq q \leq s$ and $1 \leq j \leq k_{\mu_{q}}$ such that $C=C_{j}^{\mu_{q}}$ and $X^{\lambda}(C)=X^{\mu_{q}}\left(C_{j}^{\mu_{q}}\right)$ which immediately implies that $U_{i_{0}}(\lambda, \chi)=U_{j}\left(\mu_{q}, \chi\right)$ from Lemma 3.5.2. Canceling $U_{i_{0}}(\lambda, \chi)$ and $U_{j}\left(\mu_{q}, \chi\right)$ in Equation (4.3) and proceeding by induction we get the desired the result.

We end this section with the following important corollary of Theorem 4.1.1. If we assume all the sets $\Pi(\lambda)$ involved in Theorem 4.1.1 are connected then we get the unique factorization property for $U(\lambda, \chi)$.

Corollary 4.1.2. Let $\chi: W \times Q_{+}^{\mathrm{im}} \rightarrow \mathbb{C} \backslash\{0\}$ be a homomorphism. Let $r, s \in \mathbb{N}$ and let $\bar{\lambda}=\left(\lambda_{1}, \ldots, \lambda_{r}\right) \in P_{+}^{r}, \bar{\mu}=\left(\mu_{1}, \ldots, \mu_{s}\right) \in P_{+}^{s}$ such that $\Pi\left(\lambda_{i}\right)$ and $\Pi\left(\mu_{j}\right)$ are connected for all $1 \leq i \leq r, 1 \leq j \leq s$. Suppose we have,

$$
U\left(\lambda_{1}, \chi\right) \cdots U\left(\lambda_{r}, \chi\right)=U\left(\mu_{1}, \chi\right) \cdots U\left(\mu_{s}, \chi\right)
$$

then $r=s$ and there exists a bijection $\sigma$ in $S_{r}$ such that $U\left(\lambda_{i}, \chi\right)=U\left(\mu_{\sigma(i)}, \chi\right)$ for all $1 \leq i \leq r$.

Proof. Since $\Pi(\lambda)$ are connected, we have $\mathcal{M C}(\bar{\lambda})=\left\{\Pi\left(\lambda_{i}\right): 1 \leq i \leq r\right\}$ and $\mathcal{M C}(\bar{\mu})=$ $\left\{\Pi\left(\mu_{j}\right): 1 \leq j \leq s\right\}$. Interpreting this in Theorem 4.1.1 gives us the result.

Remark 4.1.3. If we specialize Theorem 4.1.1 and its Corollary 4.1.2 to the trivial homomorphism $\chi=\mathbb{1}$ then we get the unique factorization properties of $U(\lambda, \mathbb{1}) \mathrm{s}$ as in Theorem 4.1.1 and Corollary 4.1.2. These unique factorization properties of $U(\lambda, \mathbb{1})$ are new as far as we know.

\subsection{Unique factorization of tensor products}

By specializing Theorem 4.1.1 to the sign homomorphism $\chi=$ sgn, we can determine the necessary and sufficient conditions for which the tensor product of irreducible representations from $\mathcal{O}^{\text {int }}$ is isomorphic to another.

Theorem 4.2.1. Let $\mathfrak{g}$ be a BKM algebra and let $r, s \in \mathbb{N}$ such that $r \geq s$ and let $\bar{\lambda}=$ $\left(\lambda_{1}, \ldots, \lambda_{r}\right) \in P_{+}^{r}, \bar{\mu}=\left(\mu_{1}, \ldots, \mu_{s}\right) \in P_{+}^{s}$. Set $\widetilde{\mu}=\left(\mu_{1}, \ldots, \mu_{s}, 0, \cdots, 0\right)$ where 0 appears $r-s$ times. We have,

$$
L\left(\lambda_{1}\right) \otimes L\left(\lambda_{2}\right) \otimes \cdots \otimes L\left(\lambda_{r}\right) \cong L\left(\mu_{1}\right) \otimes L\left(\mu_{2}\right) \otimes \cdots \otimes L\left(\mu_{s}\right)
$$

as $\mathfrak{g}$-modules if and only if $\sum_{i=1}^{r} \lambda_{i}=\sum_{j=1}^{s} \mu_{j}$ and there exists a bijection $\sigma: \mathcal{M C}(\bar{\lambda}) \rightarrow$ $\mathcal{M C}(\widetilde{\mu})$ given by $C \mapsto C_{\sigma}$ satisfying the following conditions: if $C \in \mathcal{M C}\left(\lambda_{i}\right)$ maps to $C_{\sigma} \in \mathcal{M C}\left(\mu_{j}\right)$ then we have 
(i) $C=C_{\sigma}$ and further, (ii) $\lambda_{i}\left(h_{\alpha}\right)=\mu_{j}\left(h_{\alpha}\right)$ for all $\alpha \in C^{\mathrm{re}}$.

Proof. Assume that (4.8) holds. Then by taking character on both sides of Equation (4.8), we get

$$
\operatorname{ch} L\left(\lambda_{1}\right) \cdots \operatorname{ch} L\left(\lambda_{r}\right)=\operatorname{ch} L\left(\mu_{1}\right) \cdots \operatorname{ch} L\left(\mu_{s}\right)
$$

By considering the maximal weights on the both sides of Equation (4.8) we get $\sum_{i=1}^{r} \lambda_{i}=$ $\sum_{j=1}^{s} \mu_{j}=: \tau$ (say). Now multiplying $e^{-\tau}$ on the both sides of the Equation (4.9) and grouping the elements $\operatorname{ch} L(\lambda) e^{-\lambda}$, we get

$$
\prod_{i=1}^{r} \operatorname{ch} L\left(\lambda_{i}\right) e^{-\lambda_{i}}=\prod_{j=1}^{s} \operatorname{ch} L\left(\mu_{j}\right) e^{-\mu_{j}}
$$

Using the Weyl-Kac character formula (i.e., the Equation (2.3)), we get

$$
U_{\lambda_{1}} \cdots U_{\lambda_{r}}=U_{\mu_{1}} \cdots U_{\mu_{s}} U_{0}^{r-s}
$$

Now the necessary condition is immediate from Theorem 4.1 .1 by specializing for $\chi=$ sgn.

For the converse part, assume that $\sum_{i=1}^{r} \lambda_{i}=\sum_{j=1}^{s} \mu_{j}$ and there exists a bijection $\sigma: \mathcal{M C}(\bar{\lambda}) \rightarrow \mathcal{M C}(\widetilde{\mu})$ satisfying (i) and (ii). It is immediate from Theorem 4.1.1 (again specialize to $\chi=$ sgn) that $U_{\lambda_{1}} \cdots U_{\lambda_{r}}=U_{\mu_{1}} \cdots U_{\mu_{s}} U_{0}^{r-s}$. Now using $\sum_{i=1}^{r} \lambda_{i}=\sum_{j=1}^{s} \mu_{j}$, we get $e^{\sum_{i=1}^{r} \lambda_{i}} U_{\lambda_{1}} \cdots U_{\lambda_{r}}=e^{\sum_{j=1}^{s} \mu_{j}} U_{\mu_{1}} \cdots U_{\mu_{s}} U_{0}^{r-s}$. Thus we get, $\prod_{i=1}^{r} \operatorname{ch} L\left(\lambda_{i}\right)=\prod_{j=1}^{s} \operatorname{ch} L\left(\mu_{j}\right)$. Since a finite tensor product of integrable irreducible modules from $\mathcal{O}$ is completely reducible ([7, Page no. 180, Corollary 10.7]), we have the isomorphism of the corresponding tensor products (4.8).

We can immediately deduce the following corollary which gives the unique factorization property for tensor products of special family of irreducible integrable representations of indecomposable BKM algebra $\mathfrak{g}$.

Corollary 4.2.2. Let $\mathfrak{g}$ be an indecomposable BKM algebra and let $r, s \in \mathbb{N}$ and let $\bar{\lambda}=$ $\left(\lambda_{1}, \ldots, \lambda_{r}\right) \in P_{+}^{r}, \bar{\mu}=\left(\mu_{1}, \ldots, \mu_{s}\right) \in P_{+}^{s}$ such that $\Pi\left(\lambda_{i}\right)$ and $\Pi\left(\mu_{j}\right)$ are connected for all $1 \leq i \leq r, 1 \leq j \leq$ s. Suppose

$$
L\left(\lambda_{1}\right) \otimes L\left(\lambda_{2}\right) \otimes \cdots \otimes L\left(\lambda_{r}\right) \cong L\left(\mu_{1}\right) \otimes L\left(\mu_{2}\right) \otimes \cdots \otimes L\left(\mu_{s}\right)
$$

as $\mathfrak{g}$-modules then $r=s$ and there exists a permutation $\sigma \in S_{r}$ such that 


$$
\operatorname{ch} L\left(\lambda_{i}\right)=e^{\lambda_{i}-\mu_{\sigma(i)}} \operatorname{ch} L\left(\mu_{\sigma(i)}\right) \text { for all } 1 \leq i \leq r .
$$

Further more if we assume that $\lambda_{i}-\mu_{\sigma(i)}$ are dominants and special for all $1 \leq i \leq r$ then there exist one-dimensional $\mathfrak{g}$-modules $Z_{i}$ such that $L\left(\lambda_{i}\right) \cong L\left(\mu_{\sigma(i)}\right) \otimes Z_{i}$ for all $1 \leq i \leq r$.

Proof. Since $\Pi\left(\lambda_{i}\right)$ and $\Pi\left(\mu_{j}\right)$ are connected for all $1 \leq i \leq r, 1 \leq j \leq s$, by Theorem 4.2.1, we get $r=s$ and there exists a permutation $\sigma \in S_{r}$ such that $\Pi\left(\lambda_{i}\right)=\Pi\left(\mu_{j}\right)$ and $\lambda_{i}\left(h_{\alpha}\right)=\mu_{\sigma(i)}\left(h_{\alpha}\right)$ for all $\alpha \in \Pi^{\mathrm{re}}$. This implies that $\lambda_{i}-\mu_{\sigma(i)}$ is $W$-invariant and we get $\operatorname{ch} L\left(\lambda_{i}\right)=e^{\lambda_{i}-\mu_{\sigma(i)}} \operatorname{ch} L\left(\mu_{\sigma(i)}\right)$ for all $1 \leq i \leq r$ from Lemma 3.5.2. The last statement immediately follows from Lemma 2.5.1.

Remark 4.2.3. We make the following important remarks:

(1) The last assumption on the dominant weights, namely that the difference is dominant and special, is needed to make sure that we get one-dimensional twists. This assumption is necessary, see the Example 4.3.1.

(2) Suppose that the Borcherds-Cartan matrix is indecomposable and $a_{i i}=2$ for all $i \in I$. Then we have $\Pi=\Pi^{\mathrm{re}}$ and $\Pi^{\mathrm{im}}=\emptyset$. Since $\Pi(\lambda)=\Pi$ is connected for all $\lambda \in P_{+}$, we recover the result for Kac-Moody algebras that was proved in [12]. This result was first proved for finite dimensional simple Lie algebras in [11].

4.3. We end with some examples.

Example 4.3.1. Suppose that the Borcherds-Cartan matrix is $\left[\begin{array}{cc}2 & -1 \\ -1 & 0\end{array}\right]$. We have $\left(\alpha_{1}, \alpha_{1}\right)=2,\left(\alpha_{1}, \alpha_{2}\right)=-1$ and $\left(\alpha_{2}, \alpha_{2}\right)=0$ where $\alpha_{1}$ and $\alpha_{2}$ are real and imaginary simple roots respectively. Note that $\mathfrak{g}$ is indecomposable (i.e., $\Pi$ is connected). The Weyl group is $W=\left\{1, \mathbf{s}_{\alpha_{1}}\right\}$. We have $\Pi(\lambda)$ is always connected for any $\lambda \in P_{+}$. Let $\lambda, \mu \in P_{+}$and write $\lambda=a \omega_{1}+b \omega_{2}, \mu=c \omega_{1}+d \omega_{2}$ where $\omega_{i}, i \in I$ are fundamental weights defined by $\omega_{i}\left(h_{j}\right)=\delta_{i j}$ for all $i, j \in I$. If $a=c$ and both $b, d$ are non-zero, then we have $\operatorname{ch} L(\lambda)=e^{\lambda-\mu} \operatorname{ch} L(\mu)$. Furthermore, $e^{\lambda-\mu}$ is not a character of a one-dimensional representation, even when $\lambda-\mu$ is dominant.

Now take $\lambda_{1}=3 \omega_{1}+4 \omega_{2}, \lambda_{2}=2 \omega_{1}+3 \omega_{2}, \mu_{1}=3 \omega_{1}+3 \omega_{2}$ and $\mu_{2}=2 \omega_{1}+4 \omega_{2}$, then using the above observation we have

$$
L\left(\lambda_{1}\right) \otimes L\left(\lambda_{2}\right) \cong L\left(\mu_{1}\right) \otimes L\left(\mu_{2}\right) \text { and } \operatorname{ch} L\left(\lambda_{i}\right)=e^{\lambda_{i}-\mu_{i}} \operatorname{ch} L\left(\mu_{i}\right), i=1,2
$$

But $\lambda_{1}-\mu_{1}=\omega_{2} \in P_{+}$is not special, thus $e^{\lambda_{1}-\mu_{1}}$ is not a character of one dimensional representation. Since $\lambda_{2}-\mu_{2}=-\omega_{2} \notin P_{+}$, it is easy to see that $e^{\lambda_{2}-\mu_{2}}$ is not a character of one dimensional representation. 
Example 4.3.2. Suppose that the Borcherds-Cartan matrix is $\left[\begin{array}{ccc}2 & -1 & 0 \\ -1 & 0 & -1 \\ 0 & -1 & 2\end{array}\right]$. Denote by $\Pi^{\mathrm{re}}=\left\{\alpha_{1}, \alpha_{2}\right\}$ and $\Pi^{\mathrm{im}}=\beta$. Note that $\left(\alpha_{1}, \alpha_{2}\right)=0,\left(\alpha_{1}, \beta\right)=\left(\alpha_{2}, \beta\right)=-1$ and $(\beta, \beta)=0$, therefore $\Pi$ is connected and $\mathfrak{g}$ is indecomposable. The Weyl group is $W=W_{1} \times W_{2}$, where $W_{i}=\left\{1, \mathbf{s}_{\alpha_{i}}\right\}$ for $i=1,2$. Let $\Gamma=\left\{\lambda \in P_{+}:(\lambda, \beta)=0\right\}$. For $\lambda \in \Gamma$, we have $\beta \in \lambda_{\mathrm{im}}^{\perp}$ and in particular $\lambda$ is a special dominant weight such that $\Pi(\lambda)=\Pi$ is connected. So, Corollary 4.2.2 applies to elements of $\Gamma$. Suppose $\mu \in P_{+} \backslash \Gamma$, then we have $\Pi(\mu)=\left\{\alpha_{1}, \alpha_{2}\right\}$ which is not connected. Write $\mu=a_{1} \omega_{1}+a_{2} \omega_{2}+a_{3} \omega_{3}$ with $a_{3} \neq 0$, where $\omega_{i}, i \in I$ are fundamental weights defined by $\omega_{i}\left(h_{j}\right)=\delta_{i j}$ for all $i, j \in I$. Since $\Omega(\mu)=0$, we have

$$
\operatorname{ch} L(\mu)=e^{\left(a_{3}+1\right) \omega_{3}} \frac{U_{a_{1} \omega_{1}}^{1} \cdot U_{a_{2} \omega_{2}}^{2}}{e^{\rho} U_{0}} \text { where } U_{a_{i} \omega_{i}}^{i}=\sum_{w \in W_{i}}(-1)^{\ell(w)} e^{w\left(a_{i}+1\right) \omega_{i}}, i=1,2 .
$$

Thus for $a_{1}, a_{2}, b_{1}, b_{2} \in \mathbb{Z}_{+}$and $a_{3}, b_{3} \in \mathbb{N}$, we get

$$
\begin{aligned}
& L\left(a_{1} \omega_{1}+a_{2} \omega_{2}+a_{3} \omega_{3}\right) \otimes L\left(b_{1} \omega_{1}+b_{2} \omega_{2}+b_{3} \omega_{3}\right) \\
& \cong L\left(a_{1} \omega_{1}+b_{2} \omega_{2}+a_{3} \omega_{3}\right) \otimes L\left(b_{1} \omega_{1}+a_{2} \omega_{2}+b_{3} \omega_{3}\right) .
\end{aligned}
$$

Remark 4.3.3. A similar phenomenon happens for typical finite-dimensional modules over Lie superalgebras. For example, take $\mathfrak{g}=\mathfrak{s l}(m \mid n)$ and $\Pi$ to be the standard choice of simple roots. Then for $\lambda$ typical, $\mathcal{M C}(\lambda)$ has two components and the character of $L(\lambda)$ factorize to two terms. However, due to the lack of complete reducibility, this is not enough to conclude about tensor products of representations. The subcategory of typical finite-dimensional $\mathfrak{g}$-modules admits complete reducibility but is not closed under tensor products.

Example 4.3.4. Suppose that $a_{i j} \in-\mathbb{Z}_{+}$for all $i, j$. Then we have $\Pi^{\mathrm{re}}=\emptyset$ and $\Pi=\Pi^{\mathrm{im}}$. Let $\lambda \in P_{+}$and let $k_{i} \in \mathbb{N}, 1 \leq i \leq r$ and $\ell_{i} \in \mathbb{N}, 1 \leq i \leq r$ such that $\sum_{i=1}^{r} k_{i}=\sum_{j=1}^{r} \ell_{j}=: s$. Then we have $\operatorname{ch}\left(L\left(k_{1} \lambda\right) \otimes \cdots \otimes L\left(k_{r} \lambda\right)\right)=e^{(s-r) \lambda} \operatorname{ch} L(\lambda)=\operatorname{ch}\left(L\left(\ell_{1} \lambda\right) \otimes \cdots \otimes L\left(\ell_{r} \lambda\right)\right)$ and hence we have $L\left(k_{1} \lambda\right) \otimes \cdots \otimes L\left(k_{r} \lambda\right) \cong L\left(\ell_{1} \lambda\right) \otimes \cdots \otimes L\left(\ell_{r} \lambda\right)$.

\section{References}

[1] G. Arunkumar, D. Kus, R. Venkatesh, Root multiplicities for Borcherds algebras and graph coloring, J. Algebra 499 (2018) 538-569.

[2] R. Borcherds, Generalized Kac-Moody algebras, J. Algebra 115 (2) (1988) 501-512.

[3] R. Borcherds, Monstrous moonshine and monstrous Lie superalgebras, Invent. Math. 109 (1992) 405-444.

[4] J.E. Humphreys, Reflection Groups and Coxeter Groups, Cambridge Studies in Advanced Mathematics, vol. 29, Cambridge University Press, Cambridge, 1990.

[5] E. Jurisich, An exposition of generalized Kac-Moody algebras, in: Lie Algebras and Their Representations, Seoul, 1995, in: Contemp. Math., vol. 194, Amer. Math. Soc., Providence, RI, 1996, pp. 121-159. 
[6] K. Jeong, S.J. Kang, M. Kashiwara, Crystal bases for quantum generalized Kac-Moody algebras, Proc. Lond. Math. Soc. 90(3) (2) (2005) 395-438.

[7] V.G. Kac, Infinite-dimensional Lie Algebras, third edition, Cambridge University Press, Cambridge, 1990.

[8] S. Naito, The strong Bernstein-Gelfand-Gelfand resolution for generalized Kac-Moody algebras, I: the existence of the resolution, Publ. RIMS, Kyoto Univ. 29 (1993) 709-730.

[9] S. Naito, Kazhdan-Lusztig conjecture for generalized Kac-Moody algebras. II: proof of the conjecture, Trans. Am. Math. Soc. 347 (10) (1995) 3891-3919.

[10] S. Naito, On the Harish-Chandra homomorphism for generalized Kac-Moody algebras, Commun. Algebra 29 (3) (2001) 1069-1084.

[11] C.S. Rajan, Unique decomposition of tensor products of irreducible representations of simple algebraic groups, Ann. Math. (2) 160 (2) (2004) 683-704.

[12] R. Venkatesh, S. Viswanath, Unique factorization of tensor products for Kac-Moody algebras, Adv. Math. 231 (6) (2012) 3162-3171. 